\title{
Modern treatment outcomes for early T-stage oropharyngeal cancer treated with intensity-modulated radiation therapy at a tertiary care institution
}

Eric J. Di Gravio ${ }^{1}$, Pencilla Lang ${ }^{2}$, Hugh Andrew Jinwook Kim ${ }^{1}$, Tricia Chinnery ${ }^{3}$, Neil Mundi ${ }^{1}$, S. Danielle MacNeil ${ }^{1,2}$, Adrian Mendez ${ }^{1,2}$, John Yoo ${ }^{1,2}$, Kevin Fung ${ }^{1,2}$, Joe S. Mymryk ${ }^{1,2,4}$, John W. Barrett ${ }^{1}$, Nancy Read ${ }^{2}$, Varagur Venkatesan ${ }^{2}$, Sara Kuruvilla², Lucas C. Mendez², Eric Winquist ${ }^{1,2}$, Sylvia Mitchell2, Sarah A. Mattonen ${ }^{2,3}$, Anthony C. Nichols ${ }^{1,2,5+}$ and David A. Palma ${ }^{1,2,5^{*}+}$

\begin{abstract}
Background: Transoral surgery (TOS), particularly transoral robotic surgery (TORS) has become the preferred modality in the United States for the treatment of early stage oropharyngeal cancer, largely due to assumptions of fewer toxicities and improved quality of life compared to primary radiotherapy (RT). However, these assumptions are based on retrospective analysis, a subset of which utilize primary RT groups not limited to T1-2 stage tumors for which transoral robotic surgery is FDA approved. Thus, there is potential for underestimating survival and overestimating toxicity, including treatment related mortality, in primary RT.
\end{abstract}

Methods: Consecutive cases of early T-stage (T1-T2) oropharyngeal cancer presenting to the London Health Sciences Centre between 2014 and 2018 treated with RT or chemoradiation (CRT) were reviewed. Patient demographics, treatment details, survival outcomes and toxicity were collected. Toxicities were retrospectively graded using the Common Terminology Criteria for Adverse Events criteria.

Results: A total of 198 patients were identified, of which $82 \%$ were male and $73 \%$ were HPV-positive. Sixty-eight percent of patients experienced a grade 2 toxicity, $48 \%$ a grade 3 and $4 \%$ a grade 4 . The most frequent toxicities were dysphagia, neutropenia and ototoxicity. The rates of gastrostomy tube dependence at 1 and 2 years were $2.5 \%$ and $1 \%$ respectively. There were no grade 5 (fatal) toxicities. HPV-positive patients experienced improved 5-year overall survival ( $86 \%$ vs $64 \%, p=0.0026)$.

Conclusions: Primary RT or CRT provides outstanding survival for early T-stage disease, with low rates of severe toxicity and feeding tube dependence. This study provides a reference for comparison for patients treated with primary transoral surgery.

Keywords: Radiation, Chemoradiation, Oropharyngeal cancer, Toxicity

*Correspondence: david.palma@lhsc.on.ca

${ }^{\dagger}$ Anthony C. Nichols and David A. Palma contributed equally to this manuscript

${ }^{5}$ Department of Otolaryngology - Head and Neck Surgery, Victoria Hospital, London Health Science Centre, 800 Commissioners Road East, London, ON N6A 5W9, Canada

Full list of author information is available at the end of the article

\section{Background}

Over the past few decades, there has been a dramatic rise in the incidence of oropharyngeal squamous cell carcinoma (OPSCC), largely due to increasing rates of infection with human papillomavirus (HPV) [1]. original author(s) and the source, provide a link to the Creative Commons licence, and indicate if changes were made. The images or other third party material in this article are included in the article's Creative Commons licence, unless indicated otherwise in a credit line to the material. If material is not included in the article's Creative Commons licence and your intended use is not permitted by statutory regulation or exceeds the permitted use, you will need to obtain permission directly from the copyright holder. To view a copy of this licence, visit http://creativecommons.org/licenses/by/4.0/. The Creative Commons Public Domain Dedication waiver (http://creativeco mmons.org/publicdomain/zero/1.0/) applies to the data made available in this article, unless otherwise stated in a credit line to the data. 
HPV-associated OPSCC patients tend to be younger and healthier than traditional smoking- and alcohol-related OPSCC patients, and have a significantly improved prognosis. The 5-year survival of HPV-associated OPSCC exceeds $80 \%$, making quality of life after treatment increasingly important since these patients may now survive for many years with significant treatment toxicities [2]. This has led to intense interest in treatment de-escalation, with the goal of reducing the toxicity of standard dose chemoradiation therapy [3]. In the United States, transoral surgery (TOS), particularly transoral robotic surgery (TORS), has largely become the preferred treatment modality for early T-stage OPSCC, as retrospective data has suggested a more favourable toxicity profile [4]. However, there is a paucity of data directly comparing the two modalities making the choice of treatment for OPSCC highly controversial [5].

The drive to adopt TOS as the standard of care revolves around the assumption that it carries a lower toxicity profile, and therefore better quality of life, than standard chemoradiation [5]. The ORATOR study, a phase II trial of 68 patients, is the only randomized clinical trial to directly compare quality of life between primary radiotherapy and primary TOS approaches, and included patients regardless of HPV status [6]. In this trial, toxicity profiles differed between the two modalities. In particular, swallowing-related quality of life as measured by the MD Anderson Dysphagia Inventory was statistically superior in the chemoradiation group as compared to the TORS group, although the difference did not represent a clinically meaningful change [6].

This discrepancy with previously published retrospective data can partially be explained by two potential sources of bias in previous studies: the inclusion of advanced stage OPSCC in chemoradiation cohorts, as well as improved toxicity profiles of modern radiation techniques $[6,7]$. TORS is only FDA approved for treatment of early stage (T1-2) OPSCC. However, many retrospective studies comparing TORS to chemoradiation include chemoradiotherapy cohorts containing advanced T-stage disease even though these patients are generally not considered candidates for TORS [4, 8-11]. Furthermore, advances in radiotherapy such as intensity-modulated radiation therapy (IMRT) allow for more conformal treatment plans, reducing prevalence and severity of side effects such as dysphagia [12-14].

The purpose of this study is to examine the modern outcomes and toxicity in patients with early stage disease (T1-2, N1-2) treated with IMRT \pm chemotherapy at a high-volume tertiary care cancer centre. We include only patients with T1-2 N1-2 disease as these patients would be considered candidates for treatment with primary transoral laser or robotic surgery.

\section{Methods}

\section{Study participants and clinical features}

Research Ethics Board approval (17222E) was obtained. A retrospective chart review was performed of all patients with early stage OPSCC treated with curativeintent radiotherapy \pm chemotherapy who presented to the London Health Sciences Centre (LHSC) between 2014 and 2018. Early stage was defined as American Joint Committee on Cancer 7th Edition stage T1-T2, N0-N2. Patient, tumour and treatment-related factors collected included: gender, age at diagnosis, smoking and alcohol history, site of primary tumour (base of tongue, palatine tonsil, soft palate, vallecula, lateral or posterior pharyngeal wall, or unknown), TNM stage, HPV status, and use of concurrent chemotherapy. HPV status was determined with p16 immunohistochemical analysis with strong and diffuse staining in $>70 \%$ of tumour cells considered positive. Patients are reported with the chemotherapy protocol that they initiated treatment with regardless of whether they completed the full course or were switched to a different protocol during treatment. Alcohol abuse was defined as a history of $>20$ alcoholic beverages per week. A significant smoking history was defined as a total of 10 or more pack-years.

\section{Radiotherapy}

All patients received definitive intent radiotherapy with IMRT with fixed-gantry or rotational techniques (tomotherapy or volumetric modulated arc therapy [VMAT]). Patients generally received 70 Gy in 35 fractions (5 daily fractions delivered per week) to the gross disease, and 56 Gy in 35 fractions to the elective nodal volume. Patients were generally treated with concurrent high dose cisplatin (100 mg/m $\mathrm{m}^{2}$ given every three weeks), excluding patients aged $\geq 70$, those with comorbidities or poor performance status or those who declined. Weekly cisplatin $\left(40 \mathrm{mg} / \mathrm{m}^{2}\right)$ could be used at the discretion of the medical oncologist. If patients were not suitable for concurrent cisplatin, alternative chemotherapy regimens could include the Calais regimen (carboplatin and 5-fluorouracil) or cetuximab. If patients did not receive concurrent systemic therapy an accelerated fractionation (6 fractions per week delivered over 5 days) was used at the discretion of the treating oncologist. Unilateral radiation was used for tonsil primaries with less than $1 \mathrm{~cm}$ extension into the tongue base or palate with $\leq 1$ ipsilateral lymph node. All other patients received bilateral treatment.

\section{Evaluation and follow-up}

After completion of radiotherapy all patients were seen at 6 weeks for a clinical assessment of response and treatment toxicity, every 3 months for the first 2 years, and then every 4 to 6 months in the third to fifth years. A 
follow-up computed tomography (CT) scan was obtained at 3 months, with additional imaging, including positron emission tomography-computed tomography (PET-CT), if there was an incomplete response or if clinically indicated. Salvage surgery was reserved for patients who had persistent disease on follow-up imaging or clinically suspicious findings. All recurrences were histologically confirmed if possible.

\section{Toxicity assessment}

Treatment toxicities were graded using the CTCAE version 5.0 [15]. Toxicities or death occurring during or within 30 days of the end of treatment were included. Toxicities assessed included dysphagia, neutropenia, febrile neutropenia, ototoxicity and acute kidney injury. Any use of nasogastric, gastrostomy (G), gastrostomyjejunostomy (GJ) tube or total parental nutrition (TPN) within this time frame was considered a grade 3 dysphagia. Any change in diet not requiring the aforementioned interventions was considered grade 2 dysphagia.

\section{Statistical analysis}

Descriptive statistics were generated for baseline patient characteristics for all patients $(\mathrm{n}=198)$. Overall survival (OS) and disease-free survival (DFS) were calculated from the date of treatment to date of recurrence (DFS), date of death, or date of last follow-up, whichever occurred first. Kaplan-Meier estimates were generated for OS and DFS stratified by HPV status and compared using the log-rank test. Univariable and multivariable Cox proportional hazards regression was performed for both OS and DFS to assess the association between baseline characteristics and OS and DFS respectively. Backward step-wise analysis was used to create the final multivariate model of overall and disease-free survival (Additional files 1 and 2). All statistical analysis was performed using the R language environment for statistical computing version 3.4.0 (open source, www.r-project.org), using two-sided statistical testing at the 0.05 significance level.

\section{Results}

\section{Patient and treatment characteristics}

A total of 198 patients (163 male and 35 female) treated with chemoradiation or radiotherapy alone were included in this analysis. Figure 1 depicts a flowchart of the screening process for inclusion. The crude median follow-up for surviving patients was 27.4 months from the completion of treatment (range: 0-63). Baseline and treatment characteristics are summarized in Table 1. Median age at diagnosis was 61 years with 114/198 patients (57.6\%) having greater than a 10 pack-year smoking history and 49/198 (24.7\%) consuming more than 20 alcoholic beverages per week. One hundred and forty-four of 198

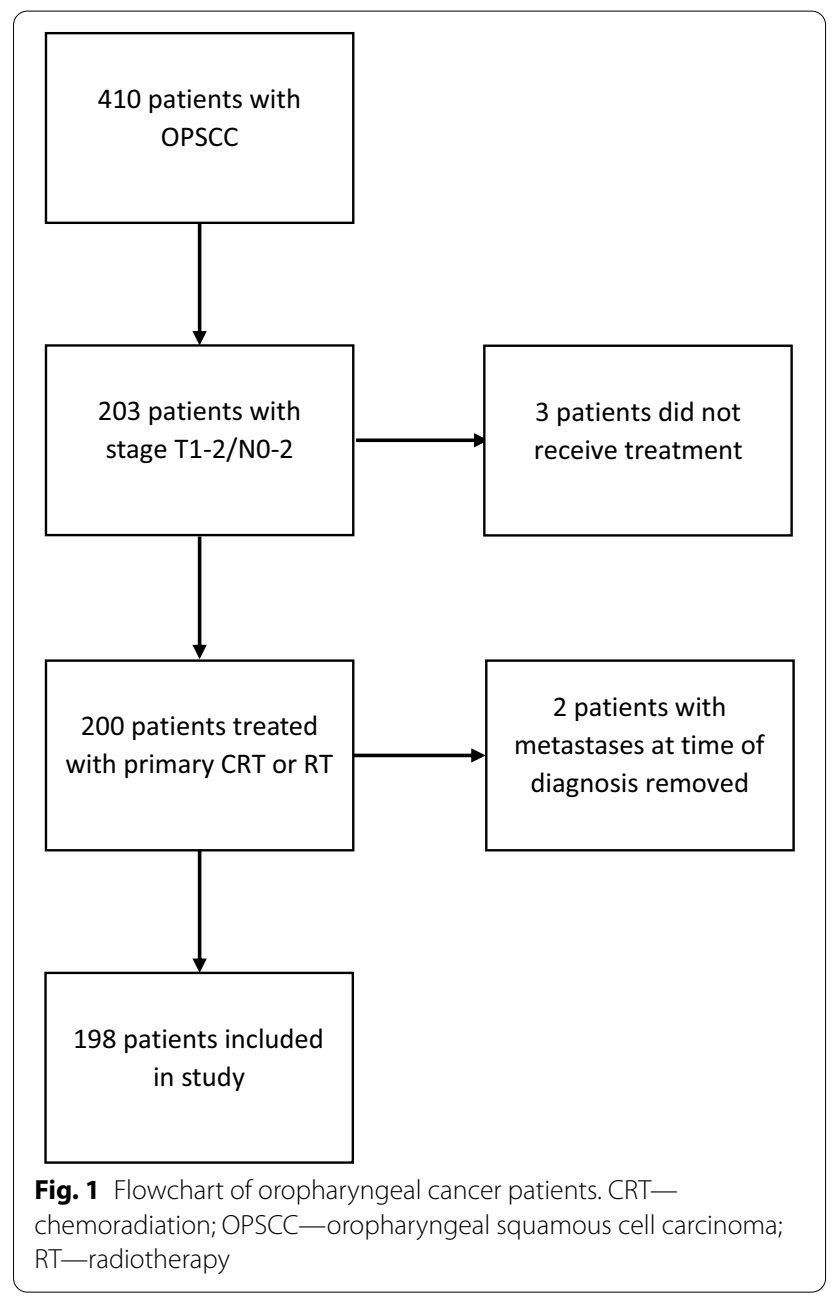

(72.7\%) patients had proven p16 positive disease and 140/198 (70.7\%) and 125/198 (63.1\%) had stage T2 and N2 disease respectively. One hundred and fifty-nine of 198 (80.3\%) patients were treated with chemoradiation with the most common chemotherapy protocol being monotherapy with cisplatin. Of patients receiving cisplatin, $61.4 \%$ received high dose treatment, while $38.5 \%$ received weekly treatment (Table 1 ). Of patients receiving high dose cisplatin, $70 / 80(87.5 \%)$ received 2 or more cycles with 54/80 (67.5\%) completing all three. Of patients treated with weekly cisplatin, $45 / 55(81.8 \%)$ completed 5 or more cycles with only $17 / 55$ (30.9\%) completing all 7 cycles.

\section{Treatment toxicity}

Data on acute toxicity is summarized in Table 2. One hundred and thirty five of 198 (68\%) of patients experienced at least one grade 2 toxicity, 95/198 (48\%) a grade 3 toxicity and $8 / 198(4 \%)$ a grade 4 toxicity. Of note, no patients experienced a grade 5 (fatal) toxicity during treatment. 
Table 1 Baseline and treatment characteristics

\begin{tabular}{|c|c|}
\hline & All patients $(n=198)$ \\
\hline Age, median (IQR) & $61(54-66)$ \\
\hline \multicolumn{2}{|l|}{ Sex } \\
\hline Male & $163(82.3 \%)$ \\
\hline Female & $35(17.7 \%)$ \\
\hline \multicolumn{2}{|l|}{ Total pack-years } \\
\hline$<10$ pack-years & $84(42.0 \%)$ \\
\hline$\geq 10$ pack-years & $114(57.6 \%)$ \\
\hline \multicolumn{2}{|l|}{ Alcohol consumption } \\
\hline$<20$ drinks per week & 149 (75.3\%) \\
\hline$\geq 20$ drinks per week & $49(24.7 \%)$ \\
\hline \multicolumn{2}{|l|}{ Primary site } \\
\hline Tonsil & $108(54.5 \%)$ \\
\hline Base of tongue & $76(38.4 \%)$ \\
\hline Soft palate & $9(4.5 \%)$ \\
\hline Vallecula & $3(1.5 \%)$ \\
\hline Indeterminate & $2(1.0 \%)$ \\
\hline \multicolumn{2}{|l|}{ Clinical T-stage } \\
\hline $\mathrm{T} 1$ & $58(29.3 \%)$ \\
\hline $\mathrm{T} 2$ & $140(70.7 \%)$ \\
\hline \multicolumn{2}{|l|}{ Clinical N-stage } \\
\hline NO & $21(10.6 \%)$ \\
\hline N1 & $52(26.3 \%)$ \\
\hline N2 & $125(63.1 \%)$ \\
\hline \multicolumn{2}{|l|}{ p16 status } \\
\hline Positive & $144(72.7 \%)$ \\
\hline Negative & $28(14.1 \%)$ \\
\hline Unknown & $26(13.1 \%)$ \\
\hline \multicolumn{2}{|l|}{ Treatment } \\
\hline $\mathrm{RT}$ & $39(19.7 \%)$ \\
\hline CRT & $159(80.3 \%)$ \\
\hline \multicolumn{2}{|l|}{ Radiation laterality } \\
\hline Unilateral & $14(7.1 \%)$ \\
\hline Bilateral & $163(82.3 \%)$ \\
\hline Unknown & $21(10.6 \%)$ \\
\hline \multicolumn{2}{|l|}{ CT simulation } \\
\hline With contrast & 85 (42.9\%) \\
\hline Without contrast & $92(46.5 \%)$ \\
\hline Unknown & $21(10.6 \%)$ \\
\hline \multicolumn{2}{|l|}{ Chemotherapy regimen } \\
\hline Cisplatin & $135(68.2 \%)$ \\
\hline High dose & $83(61.4 \%)$ \\
\hline Weekly & $52(38.5 \%)$ \\
\hline Carboplatinum + 5-fluorouracil & $14(7.1 \%)$ \\
\hline Cetuximab & $5(2.5 \%)$ \\
\hline Other & $5(2.5 \%)$ \\
\hline
\end{tabular}

Data are presented as number (\%) unless otherwise specified

$R T$ radiotherapy only, $C R T$ chemoradiation
The most common toxicity was dysphagia followed by neutropenia and ototoxicity. Patients treated with radiation alone experienced fewer toxicities compared to patients treated with chemoradiation $(\mathrm{p}<0.0001)$. Most notably, in total, 56/198 (28\%) of patients experienced grade 3 dysphagia and 37/198 (19\%) required placement of G/GJ tube. However, the rate of G/GJ tube use during treatment was significantly greater in patients treated with chemoradiation compared to patients treated with radiation alone $(\mathrm{p}=0.0196)$. Among patients treated with radiation alone, rates of grade 3 dysphagia and G/ GJ tube insertion during treatment were $4 / 39(10 \%)$ and $2 / 39(5.1 \%)$ respectively, compared to $52 / 159$ (33\%) and $33 / 159$ (21\%) for patients treated with chemoradiation. Two patients ( 1 treated with radiation alone and the other with chemoradiation) required G/GJ tube placement more than a month after completion of treatment. In total, the 1- and 2-year rates of G/GJ tube use were 2.5 and $1 \%$ respectively ( 5 and 2 patients respectively). Only 8 patients (4\%) experienced a grade 4 toxicity ( 2 ototoxicity, 6 neutropenia).

\section{Predictors of overall and disease-free survival}

In total, 32/198 (16\%) patients included in this study died. Seventeen of these 32 (53\%) patients died with disease still present, either as a direct result of their disease or due to unrelated causes, while $15 / 32$ (47\%) died of unrelated causes while in remission. Furthermore, 32/198 (16\%) of patients experienced disease recurrence, of which $27 / 32$ (84\%) were histopathologically proven. Nine of $32(28 \%)$ recurrences were local, $13 / 32(41 \%)$ were regional, $8 / 32(25 \%)$ were distant, $1 / 32(3 \%)$ was both local and regional and 1/32 (3\%) was both regional and distant. Nineteen of 32 (59\%) patients with recurrence required salvage surgery.

Thirty-two of 198 patients with incomplete clinical information were omitted from Cox proportional hazards regression. Univariable analysis revealed age, smoking status, alcohol use and p16- status as significant prognostic factors for OS (Additional file 1). With multivariable analysis, smoking history (HR: 3.59 95\% CI: $1.02-12.7, \mathrm{p}=0.0472$ ) remained a significant prognostic factor for OS (Additional file 1). Age and alcohol use were predictors of DFS in univariable and multivariable analysis (Additional file 2). p16-positive status was associated with improved OS $(\mathrm{p}=0.0026)$ but not DFS $(\mathrm{p}=0.17$, Fig. 2).

\section{Patterns of treatment failure}

Disease relapse stratified by p16 status is outlined in Fig. 3. Consistent with the literature, p16-negative patients were more likely to experience locoregional relapse than p16-positive patients (7/28 vs. $10 / 144$, 
Table 2 Summary of toxicities

\begin{tabular}{|c|c|c|c|c|c|c|c|c|}
\hline \multirow[b]{2}{*}{ Toxicity } & \multicolumn{4}{|c|}{ Radiotherapy group $(n=39)$} & \multicolumn{4}{|c|}{ Chemoradiation group $(n=159)$} \\
\hline & Grade 1-2 & Grade 3 & Grade 4 & Grade 5 & Grade 1-2 & Grade 3 & Grade 4 & Grade 5 \\
\hline Dysphagia & $26(67 \%)$ & $4(10 \%)$ & 0 & 0 & $99(62 \%)$ & $52(33 \%)$ & 0 & 0 \\
\hline Neutropenia & 0 & 0 & 0 & 0 & $64(40 \%)$ & $34(21 \%)$ & $6(4 \%)$ & 0 \\
\hline Febrile neutropenia & 0 & 0 & 0 & 0 & 0 & $14(9 \%)$ & 0 & 0 \\
\hline Ototoxicity & $1(3 \%)$ & & & 0 & $46(29 \%)$ & $34(21 \%)$ & $2(1 \%)$ & 0 \\
\hline Acute kidney injury & 0 & 0 & 0 & 0 & 0 & $10(6 \%)$ & 0 & 0 \\
\hline
\end{tabular}

Data are presented as numbers (\%). Grading is consistent with Common Terminology Criteria for Adverse Events (CTCAE) version 5.0

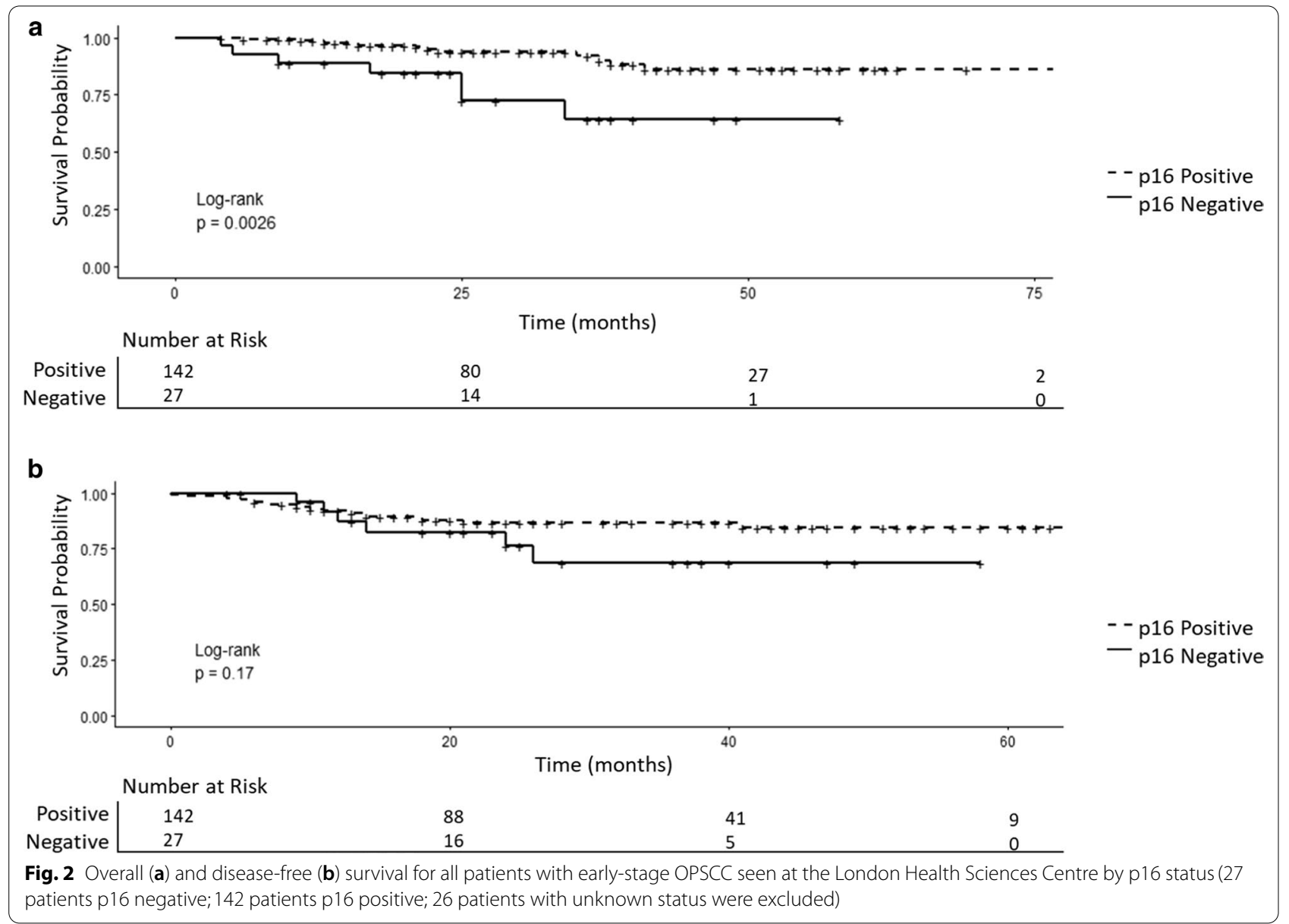

$\mathrm{p}<0.01)$, and significantly more likely to die from that recurrence $(7 / 7$ vs. $3 / 10, p=0.0089)$ [3]. There were nine distant metastatic failures in the p16-positive cohort including lesions in the lung, liver and skeleton, while none occurred in the p16-negative group, however this was not statistically significant $(9 / 144$ vs. $0 / 28$, $\mathrm{p}=0.36$ ).

\section{Discussion}

In this study, we demonstrated that while IMRT-based (chemo)radiation can have short- and long-term toxicities, early-stage HPV-associated OPSCC patients experienced excellent survival with acceptable toxicities, low long-term gastrostomy dependency rates and negligible treatment-related mortality ( $0 \%$ in this study). In 


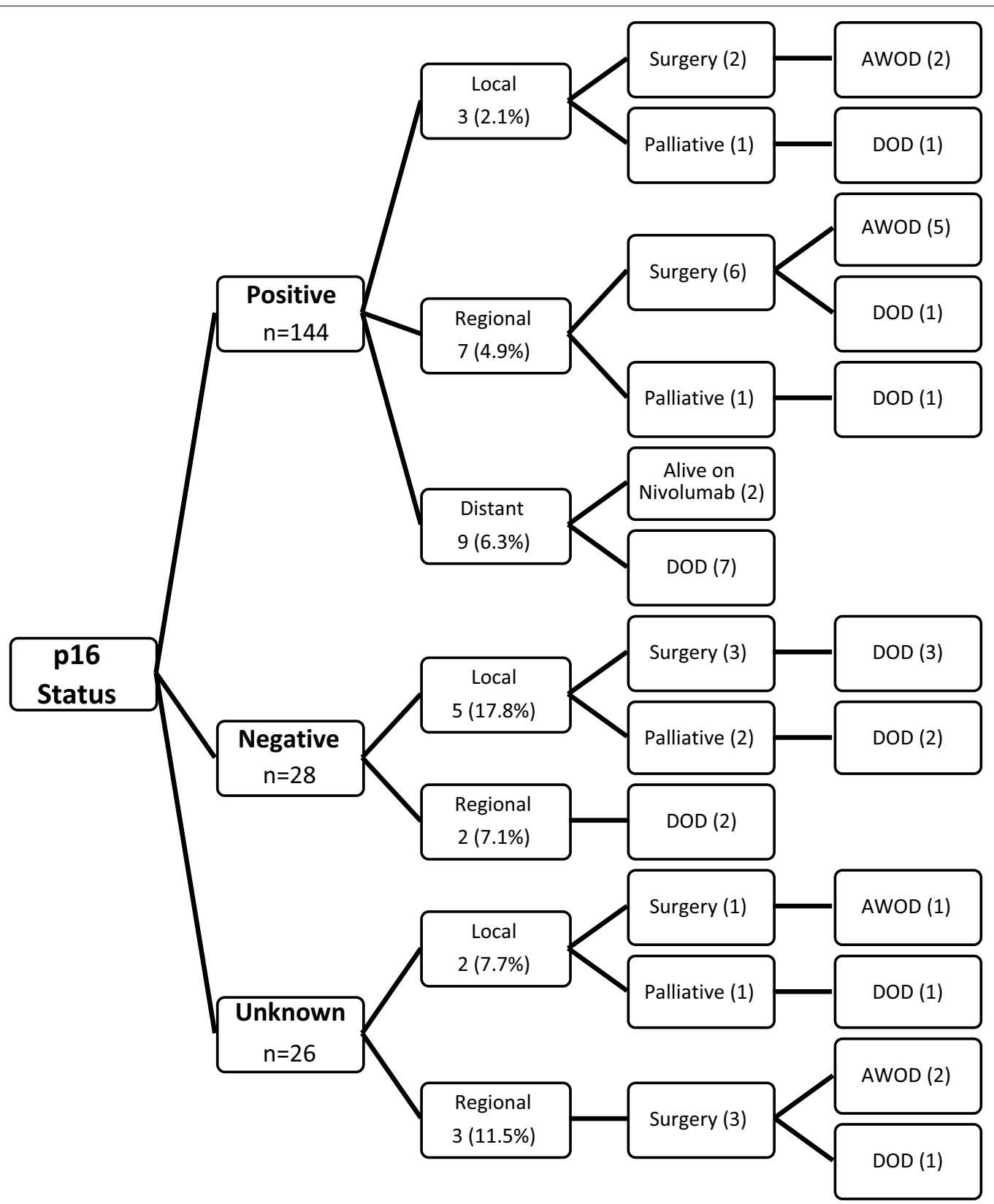

Fig. 3 Patterns of treatment failure stratified by p16 status (positive, negative or unknown). AWOD—alive without disease; DOD—dead of disease

contrast, prior studies have reported higher toxicity rates, with treatment related deaths of up to $3 \%[2,16]$. Similarly, a frequently cited meta-analysis of the Radiation Therapy Oncology Group treatment intensification trials by Machtay and colleagues reported that $43 \%$ of patients treated with primary chemoradiation for OPSCC suffered a severe major late toxicity, including a $10 \%$ rate of long-term feeding tube dependency [16]. This is relevant as these studies are often cited as a reference comparison for primary transoral surgery $[4,8-11]$. While TOS can be carried out for advanced stage disease, in most case series, $>85 \%$ of tumours are limited T1-T2 stage [4, $8-10]$. In contrast, the chemoradiation studies included high rates of advanced T stage disease $(>75 \%$ in one such study by Ang and colleagues) [3], introducing bias into the comparison of historical series. Furthermore, many of these studies were carried out in the pre-IMRT era, which would likely impact function and toxicity $[2,13$, $14,16]$. Patients eligible for TOS have low-volume disease that may allow for unilateral radiotherapy and improved 
normal tissue sparing compared to historical chemoradiation cohorts that include a wider range of patients. There is a deficiency in the literature of reported outcomes of solely early-stage patients. This study reports the outcomes and toxicities of early-stage oropharynx patients undergoing primary radiotherapy with modern techniques, and provides a historical comparison cohort for discussions around surgical outcomes. Rates of toxicity and treatment-related mortality are lower than in previously published cohorts.

Febrile neutropenia is a potentially life threatening and frequent complication of chemoradiation in many studies $[17,18]$. For example, a study by Bledsoe and colleagues reported a rate of febrile neutropenia of $26 \%$ in patients treated with chemoradiation with $2 / 32(6.3 \%)$ of these patients dying as a direct result [17]. In contrast, 19.7\% of our early-stage patients did not require chemotherapy at all and thus were not at risk of this complication. In the chemoradiation cohort specifically, the rate of febrile neutropenia was only $8.8 \%$ and there were no fatalities (Table 2).

One of the strongest predictors of poor patient quality of life following treatment for head and neck cancer is long-term gastrostomy tube dependence [19]. Chemoradiation studies report rates of up to $10 \%$ long-term dependence while most TOS studies show lower rates $[4$, $20,21]$. In a previous systematic review, the majority of studies demonstrated gastrostomy rates of less than $4.5 \%$, with many (6/13) showing rates of $0 \%$ suggesting superior swallowing function with primary surgery [4]. However, this study has 2-year gastrostomy rates of $1 \%$. This is similar to the results of the ORATOR trial, and suggest that swallowing outcomes are similar between the two treatment strategies [6]. Given the similar survival, treatment selection for early OPSCC should be an informed decision made between clinicians and patients.

In this study, we also attempt to describe potential prognostic indicators for OS and DFS. It is well-established that HPV-associated OPSCC has a significantly better OS and DFS than HPV-negative patients [3]. In our study, OS but not DFS was found to be significantly better in HPV-associated OPSCC, possibly due to limited sample size (Fig. 1). In early stage disease, chemoradiation appeared effective regardless of HPV status, and the differences in OS may be partially related to the presumed increased incidence of comorbidities in HPV-negative patients and subsequent non-cancer related death. Likely for similar reasons, on multivariable analysis, a history of smoking was associated with a worse OS but not DFS (Additional files 1 and 2).

Concurrent chemoradiation with 3 cycles of highdose cisplatin is currently the standard treatment for locoregionally advanced OPSCC [22]. However, due to short- and long-term toxicities, and due to the fact that HPV-associated OPSCC is more sensitive to chemotherapy and radiation than HPV-negative OPSCC, there has been much interest in treatment de-escalation [23, 24]. Some current strategies under investigation include weekly cisplatin instead of high-dose cisplatin, lower radiation dose, or decreased adjuvant radiation and/ or chemotherapy after surgery [25-27]. Other strategies, such as using the epidermal growth factor receptor (EGFR) monoclonal antibody cetuximab, have conclusively been shown to provide inferior survival without meaningfully improving quality of life which only further highlights the importance of balancing toxicity with survival $[28,29]$.

There are a number of limitations with this current study. First of all, it is inherently difficult to accurately grade toxicities retrospectively, precluding the inclusion of other common but less severe toxicities such as mucositis, xerostomia or peripheral neuropathy. The follow-up period was limited and thus may have led to an underestimation of long-term toxicities. This data is limited to a single centre and thus may not accurately portray the true variability in the patient population. Lastly, a number of important potential confounders such as socioeconomic factors and compliance with treatment were not addressed.

\section{Conclusions}

Patients with early-stage OPSCC have excellent survival, and current assumptions about the toxicities associated with chemoradiation are likely over-stated in this population. Early-stage patients have low rates of gastrostomy tube dependence and treatment-related mortality. The lower rate of toxicity in early-stage patients compared to historical series is important to keep in mind when comparing toxicity profiles between chemoradiation and TOS. This study can provide a reference for comparison for patients treated with primary transoral laser or robotic surgery in future trials.

\section{Supplementary information}

Supplementary information accompanies this paper at https://doi. org/10.1186/s13014-020-01705-1.

Additional file 1. Univariable and multivariable analysis of overall survival in all patients with early stage disease seen at the London Health Sciences Centre from 2014 to 2018 by clinical characteristics. Samples missing clinical information were excluded (30 patients were excluded, 168 remaining samples in the analysis). Backwards step-wise method was utilized to arrive at the final multivariate model that was based on patient age, alcohol abuse, and smoking status. P-values $<0.05$ are bolded. HRhazard ratio; $\mathrm{Cl}$ —confidence interval; BoT_-base of tongue; HPV—human papillomavirus.

Additional file 2. Univariable and multivariable analysis of disease-free survival in all patients with early stage disease seen in the London Health 
Sciences Centre from 2014 to 2018 by clinical characteristics. Samples missing clinical information were excluded (32 patients were excluded, 166 remaining samples in the analysis). Backwards step-wise method was utilized to arrive at the final multivariate model that was based on patient age, sex, and alcohol abuse. P-values $<0.05$ are bolded. HR-hazard ratio; $\mathrm{Cl}$ —confidence interval; BoT—base of tongue; HPV—human papillomavirus.

\section{Abbreviations}

CRT: Chemoradiation; CTCAE: Common terminology criteria for adverse events; DFS: Disease free survival; HPV: Human papillomavirus; IMRT: Intensity modulated radiotherapy; LHSC: London Health Sciences Centre; OPSCC: Oropharyngeal squamous cell carcinoma; OS: Overall survival; RT: Radiotherapy; TORS: Transoral robotic surgery; TOS: Transoral surgery; VMAT: Volumentric modulated arc therapy.

\section{Acknowledgements}

Not applicable.

\section{Authors' contributions}

EJD, ACN and DAP designed the study. EJD, PL, HAJK, TC, SM and ACN performed the analyses. All authors read and approved the final manuscript. EJD and ACN had full access to all the data in the study and takes responsibility for the integrity of the data and the accuracy of the data analysis. All authors read and approved the final manuscript.

\section{Funding}

This work was supported by Canadian Institutes of Health Research Grant MOP\#142491 to JSM and ACN. ACN was supported by the Wolfe Surgical Research Professorship in the Biology of Head and Neck Cancers Fund.

\section{Availability of data and materials}

All data generated and analyzed during this study are included in this published article (and its supplementary information files).

\section{Ethics approval and consent to participate}

Research Ethics Board approval (17222E) was obtained.

\section{Consent for publication}

Not applicable.

\section{Competing interests}

The authors declare that they have no competing interests.

\section{Author details}

${ }^{1}$ Department of Otolaryngology - Head and Neck Surgery, Western University, London, ON, Canada. ${ }^{2}$ Department of Oncology, Western University, London, ON, Canada. ${ }^{3}$ Department of Medical Biophysics, Western University, London, ON, Canada. ${ }^{4}$ Department of Microbiology and Immunology, Western University, London, ON, Canada. ${ }^{5}$ Department of Otolaryngology - Head and Neck Surgery, Victoria Hospital, London Health Science Centre, 800 Commissioners Road East, London, ON N6A 5W9, Canada.

Received: 22 September 2020 Accepted: 30 October 2020 Published online: 10 November 2020

\section{References}

1. Chaturvedi AK, Anderson WF, Lortet-Tieulent J, et al. Worldwide trends in incidence rates for oral cavity and oropharyngeal cancers. J Clin Oncol. 2013:31:4550-9.

2. Ringash J. Survivorship and quality of life in head and neck cancer. J Clin Oncol. 2015;33:3322-7.

3. Ang KK, Harris J, Wheeler R, et al. Human papillomavirus and survival of patients with oropharyngeal cancer. N Engl J Med. 2010;362:24-35.
4. Yeh DH, Tam S, Fung K, et al. Transoral robotic surgery vs radiotherapy for management of oropharyngeal squamaous cell carcinoma: a systematic review of the literature. Eur J Surg Oncol. 2015;41:1603-14.

5. Cracchiolo JR, Baxi SS, Morris LG, et al. Increase in primary surgical treatment of 11 and $\mathrm{T} 2$ oropharyngeal squamous cell carcinoma and rates of adverse pathologic features: National Cancer Data Base. Cancer. 2016;122:1523-32.

6. Nichols AC, Theurer J, Prisman E, et al. Radiotherapy versus transoral robotic surgery and neck dissection for oropharyngeal squamous cell carcinoma (ORATOR): an open-label, phase 2, randomised trial. Lancet. 2019;20:1349-59.

7. de Almeida JR, Byrd JK, Wu R, et al. A systematic review of transoral robotic surgery and radiotherapy for early oropharynx cancer: a systematic review. Laryngoscope. 2014;124:2096-102.

8. Weinstein GS, O'Malley BW, Magnuson JS, et al. Transoral robotic surgery: a multicenter study to assess feasibility, safety and surgical margins. Laryngoscope. 2012;122:1701-7.

9. Weinstein GS, O'Malley BW, Cohen MA, et al. Transoral robotic surgery for advanced oropharyngeal carcinoma. JAMA. 2010;136:1079-85.

10. Moore EJ, Olsen SM, Laborde RR, et al. Long-term functional and oncological results of transoral robotic surgery for oropharyngeal squamous cell carcinoma. Mayo Clin Proc. 2012;87:210-25.

11. Holsinger FC, Ferris RL. Transoral endoscopic head and neck surgery and its role within the multidisciplinary treatment paradigm of oropharynx Cancer: robotics, lasers and clinical trials. J Clin Oncol. 2015;33:3285-92.

12. Gupta T, Kannan S, Ghosh-Laskar S, Agarwal JP. Systematic review and meta-analyses of intensity-modulated radiation therapy versus conventional two-dimensional and/or or three-dimensional radiotherapy in curative-intent management of head and neck squamous cell carcinoma. PLOS ONE. 2018;13:e0200137.

13. Ursino S, Dngelo E, Mazzola R, et al. A comparison of swallowing dysfunction after three-dimensional conformal and intensity-modulated radiotherapy: a systematic review by the Italian Head and Neck Radiotherapy Study Group. Strahlenther Onkol. 2017;193:877-89. https://doi. org/10.1007/s00066-017-1160-7.

14. Mazzola R, Ferrera G, Alongi F, et al. Organ sparing and clinical outcome with step-and-shoot IMRT for head and neck cancer: a mono-institutional experience. Radiol Med. 2015;120:753-8. https://doi.org/10.1007/s1154 7-015-0512-6.

15. U.S. Department of Health and Human Services, National Institutes of Health, National Cancer Institute. Common terminology criteria for adverse events (CTCadverse event) version 5.0. 2017.

16. Machtay M, Moughan J, Trotti A, et al. Factors associated with severe late toxicity after concurrent chemoradiation for locally advanced head and neck cancer: an RTOG analysis. J Clin Oncol. 2008;26:3582-9.

17. Bledsoe TJ, Noble AR, Hunter GK, et al. Oropharyngeal squamous cell carcinoma with known human papillomavirus status treated with definitive chemoradiotherapy: patterns of failure and toxicity outcomes. Radiat Oncol. 2013. https://doi.org/10.1186/1748-717X-8-174.

18. Urban D, Corry J, Solomon B, et al. Weekly cisplatin and radiotherapy for low risk, locoregionally advanced human papillomavirus-positive oropharyngeal squamous cell carcinoma. Head Neck. 2015;38:E1117-21.

19. Wells M, Swartzman S, Lanh $H$, et al. Predictors of quality of life in head and neck cancer survivors up to 5 years after end of treatment: a crosssectional survey. Support Care Cancer. 2016;24:2463-72.

20. Chen AM, Daly ME, Luu Q, et al. Comparison of functional outcomes and quality of life between transoral surgery and definitive chemoradiotherapy for oropharyngeal cancer. Head Neck. 2014;37:382-5.

21. Sharma A, Patel S, Baik FM, et al. Survival and gastrostomy prevalence in patients with oropharyngeal cancer treated with transoral robotic surgery vs. chemoradiotherapy. JAMA Otolaryngol Head Neck Surg. 2016;142:691-7.

22. You EL, Henry M, Zeitouni AG. Human papillomavirus-associated oropharyngeal cancer: review of current evidence and management. Curr Oncol. 2019;26:119-23.

23. Oosthuizen JC, Doody J. De-intensified treatment in human papillomavirus-positive oropharyngeal cancer. Lancet. 2019;393:5-7.

24. Price KAR, Nichols AC, Shen CJ, et al. Novel strategies to effectively de-escalate curative-intent therapy for patients with HPV-associated oropharyngeal cancer: current and future directions. Am Soc Clin Oncol Educ Book. 2020;40:1-13. 
25. Deschuymer S, Mahanna H, Nuyts S. Toxicity reduction in the treatment of HPV positive oropharyngeal cancer: emerging combined modality approaches. Front Oncol. 2018;8:439.

26. Hargreaves S, Beasley M, Hurt C, et al. Deintensification of adjuvant treatment after transoral surgery in patient with human papillomaviruspositive oropharyngeal cancer: the conception of the PATHOS study and its development. Front Oncol. 2019;9:936.

27. Nichols AC, Lang P, Prisman E, et al. Treatment de-escalation for HPVassociated oropharyngeal squamous cell carcinoma with radiotherapy vs. trans-oral surgery (ORATOR2): study protocol for a randomized phase II trial. BMC Cancer. 2020;20:125

28. Mehanna H, Robinson M, Hartley A, et al. Radiotherapy plus cisplatin or cetuximab in low-risk human papillomavirus-positive oropharyngeal cancer (De-ESCALaTE HPV): an open-label randomised controlled phase 3 trial. Lancet. 2019;393:51-60.

29. Gillison ML, Trotti AM, Harris J, et al. Radiotherapy plus cetuximab or cisplatin in human papillomavirus-positive oropharyngeal cancer (NRG oncology RTOG 1016): a randomised, multicentre, non-inferiority trial. Lancet. 2019;393:40-50.

\section{Publisher's Note}

Springer Nature remains neutral with regard to jurisdictional claims in published maps and institutional affiliations.
Ready to submit your research? Choose BMC and benefit from:

- fast, convenient online submission

- thorough peer review by experienced researchers in your field

- rapid publication on acceptance

- support for research data, including large and complex data types

- gold Open Access which fosters wider collaboration and increased citations

- maximum visibility for your research: over $100 \mathrm{M}$ website views per year

At BMC, research is always in progress.

Learn more biomedcentral.com/submissions 